\title{
Mathematical Model of Blast Furnace with Radially Distributed Burdens*
}

\author{
By Mamoru KUWABARA $A^{* *}$ and Iwao MUCHI**
}

\section{Synopsis}

A mathematical model of the blast furnace with the inclined layered ore and coke burdens has been developed over the region from the top to the melting level of the furnace. The thickness of each layer was assumed to change in the radial direction.

$B y$ the use of this model, the longitudinal and radial distributions of the process variables in a blast furnace operated in Japan, B.F. (DII), have been analyzed with the aid of a digital computer. The process variables include temperature, gas composition, gas velocity, and fractional reduction of iron ore.

It has been found from the calculated results that the radial distributions of the process variables mentioned above and the melting level are remarkably uneven in B.F. (DII). The present results agree with the results of the measurements by the probes or those of the dissection of the "quenched" commercial furnaces observed by the other investigators.

\section{Introduction}

Because the process variables in the blast furnace distribute in the radial direction as well as in the longitudinal direction, it is important to clarify the relationship between the inner features introduced by the radially distribution of process variables and the operating conditions. The result will increase the productivity by applying the optimum operating conditions.

Recently, to detect the inner features in the operating furnace, several investigations have been performed by inserting the probes ${ }^{1-8}$ ) into the furnace and by dissecting $^{9-14)}$ the quenched furnace. The latter investigations have shown us the descending behaviors of the layered ore and coke burdens ${ }^{10,11,14)}$ and also the location and the shape of the melting zone. ${ }^{13,14}$ However, to explain quantitatively the relationship between these observed results and the operating conditions, many problems are still to be solved.

On the other hand, to estimate the features in the region from the top to the melting level of blast furnace, Lahiri and Seshadri ${ }^{15)}$ developed a mathematical model composed of seven simultaneous partial differential equations by assuming the stack as a cylindrical reactor. They took account of the diffusion of gas in radial direction and the heat conduction through the burdens. Their study gave the radial distributions of the temperature and the composition of gas on the basis of the initial conditions concerning the radial distributions of the temperature, the composition and the flow rate of gas at the top of bed. However, the validity of their model would have limitations, because of their hypothetical radial distributions of burdens. Also their assumption of the equal temperatures for the ore and coke burdens would not be realistic at higher temperature regions. The long time needed for the numerical computation seems also disadvantageous.

The other theoretical study dealing with the radial distribution problem was reported by Chon and Tate. ${ }^{17)}$ They proposed a model in which the blast furnace was divided into three coaxial annular zones. By the use of the model, the effects of the uneven distribution of the burdens on the operating results were examined in an experimental furnace.

However, their results seemed not good enough to have a definite conclusion on the problem. Therefore, in the present paper, the relationship between the peculiar distributions of burdens to their charging method and the resultant radial distributions of the flow rate of gas is firstly considered. Then, a new model of the furnace with inclined layers of burdens is developed, applying some modifications to the previous mathematical model ${ }^{16}$ ) of the furnace with horizontally layered burdens. Furthermore, the numerical computations have been performed to predict the longitudinal and radial distributions of the process variables in the blast furnace operating in Japan.

\section{Radial Distribution of Flow Rate of Gas}

Polthier ${ }^{18)}$ has measured the radial distributions of the velocities of gas flowing through the cylindrical packed beds having the V-shaped or M-shaped top contour of the burdens. He has presented a simple expression for the profiles of gas velocity in such beds on the assumptions of the uniform voidage throughout the bed and the piston flow of gas. The other assumptions made are that the flow rate of gas might be radially distributed so as to maintain the same total pressure drop at every radial position independent of the differences in the bed heights. According to this assumption the gas pressure distributed radially may be higher in the peripheral region than in the central region at any level of bed. However, the observations by the other investigators show that the radial distribution of the gas pressure is either even ${ }^{4,19)}$ except the region near the top of the bed or almost reverse ${ }^{1)}$ in comparison with the tendency as mentioned above.

The present authors considered that the radial velocity profiles of gas may result from the radial distribution of the resistance to gas flow caused by the radial variation in each thickness of the ore and coke layers. Such effect of the contour of the bed as mentioned by Polthier would be relatively insignificant.

* Originally published in Tetsu-to-Hagané, 61 (1975), 787, in Japanese. English version received July 17, 1976.

** Department of Iron and Steel Engineering, Nagoya University, Furo-cho, Chikusa-ku, Nagoya 464. 


\section{Estimation of Radial Distribution of Flow Rate of Gas}

Before making the model, the following assumptions are made:

(1) As shown in Fig. 1, the layered coke and ore burdens charged to the top of the furnace are descending with forming the $\mathrm{V}$-shaped contours whose apparent angles of repose are $\alpha_{1}$ and $\alpha_{2}$, respectively.

(2) When the two adjacent layers are considered as one block, the $i$-th block is a cylindrically packed bed having the average diameter of $R_{i}$, and the resistance to flow at a given dimensionless radial position $\left(\zeta=r / R_{i}\right)$ is the same in any block.

(3) Ore and coke burdens are prepared through a sufficient screening, and consequently there exist no radial distributions of the particle size $d_{p j}$ and the voidage $\varepsilon_{j}$ in each layer.

With regard to any one block, taking account of the radial distribution of the longitudinally averaged particle size $\bar{d}_{p}$ and the voidage $\bar{\varepsilon}$, Ergun's equation ${ }^{20)}$ expressing the pressure drop of gas may be rewritten as follows:

$$
\partial P / \partial l=\left(\rho / g_{c}\right)\{1-\bar{\varepsilon}(\zeta)\} \cdot \bar{\Psi}(\zeta) \cdot u(\zeta)^{2} /\left\{\bar{\phi}(\zeta) \cdot \bar{d}_{p}(\zeta) \cdot \bar{\varepsilon}(\zeta)\right\} \ldots
$$

where the drag coefficient $\bar{\Psi}(\zeta)$ is defined by Eq. (2).

$$
\begin{aligned}
& \bar{T}(\zeta) \equiv 150\{1-\bar{\varepsilon}(\zeta)\} / \operatorname{Re}_{p}(\zeta)+1.75 \\
& \operatorname{Re}_{p}(\zeta) \equiv \bar{\phi}(\zeta) \cdot d_{p}(\zeta) \cdot \bar{\varepsilon}(\zeta) \cdot u(\zeta) / \nu \quad \ldots \ldots
\end{aligned}
$$

Because the value of $R e_{p}$ is very large in a blast furnace, the radial change in $\bar{\psi}(\zeta)$ becomes slight even if there were the radial changes in $d_{p j}$ and $\varepsilon_{j}$ in each layer. Consequently, it may be assumed as $\bar{\Psi}(\zeta) \equiv \Psi$ (constant). Moreover, it is assumed that the shape factor may have a constant value ${ }^{16)}$ and is expressed by $\bar{\phi}(\zeta)=\phi_{1}=\phi_{2}=0.64 \equiv \phi$.

Each volume of the coke and the ore burdens in a block which is enclosed between the radial position $\zeta$ and $(\zeta+d \zeta)$ are designated by $V_{1 \zeta}$ and $V_{2 \zeta}$, respec-

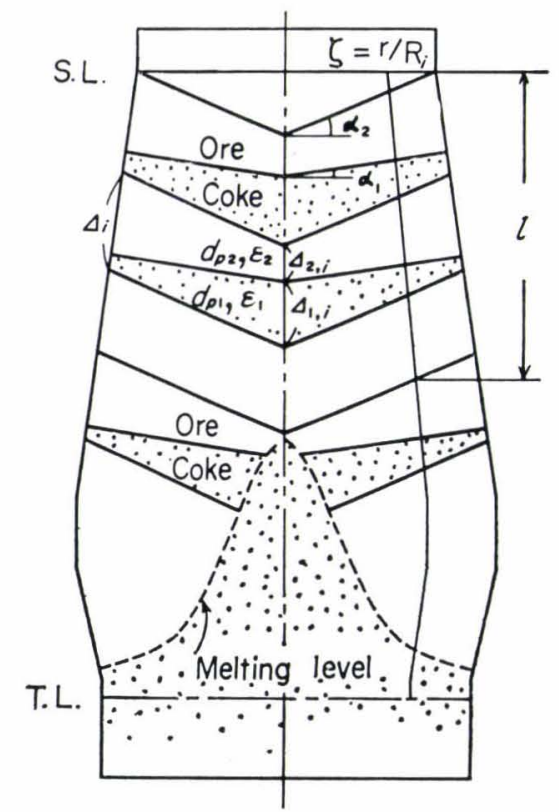

Fig. 1. Schematic diagram of blast furnace with radially distributed burdens tively. Then, by using these volumes, $\bar{\varepsilon}(\zeta)$ can be readily expressed as:

$$
\bar{\varepsilon}(\zeta)=\left(\varepsilon_{1} V_{1 \zeta}+\varepsilon_{2} V_{2 \zeta}\right) /\left(V_{1 \zeta}+V_{2 \zeta}\right)=A+B \zeta
$$

where the dimensionless factors, $A$ and $B$, are related to the charging conditions through the following expressions:

$$
\begin{aligned}
A & \equiv\left\{\varepsilon_{1} V_{1}+\varepsilon_{2} V_{2}+(2 / 3) \pi R_{1}^{3} A\left(\varepsilon_{1}-\varepsilon_{2}\right)\right\} /\left(V_{1}+V_{2}\right) \\
B & \equiv-\pi R_{1}^{3} A\left(\varepsilon_{1}-\varepsilon_{2}\right) /\left(V_{1}+V_{2}\right) \\
A & \equiv \tan \alpha_{2}-\tan \alpha_{1} \ldots \ldots \ldots \ldots \ldots \ldots \ldots \ldots \ldots \ldots \ldots \ldots \ldots \ldots \ldots \ldots \ldots \ldots \ldots \ldots
\end{aligned}
$$

Mean particle size $d_{p}(\zeta)$ should be determined so as to meet the stipulation that the pressure drop of the gas through a certain block having $\bar{\varepsilon}(\zeta)$ and $d_{p}(\zeta)$ must be equated to the sum of that through each layer in the block. This may imply that $\Delta_{i} /\left\{2 \phi \bar{d}_{p} \bar{\varepsilon} \mid\right.$ $3(1-\bar{\varepsilon})\}$ is expressed as the sum of $J_{j} /\left\{2 \phi_{j} d_{p j} \varepsilon_{j} / 3\left(1-\varepsilon_{j}\right)\right\}$ in each layer. Consequently, we have

$$
\bar{d}_{p}(\zeta)=\Delta_{i}\{1-\bar{\varepsilon}(\zeta)\} /\left\{\bar{\varepsilon}(\zeta) \cdot\left(C_{i}+D_{i} \zeta\right)\right\}
$$

where $\lrcorner_{i}, C_{i}$ and $D_{i}$ are represented by Eq. (6).

$$
\begin{aligned}
& J_{i}=\left(V_{1}+V_{2}\right) /\left(\pi R_{i}^{2}\right) \\
& C_{i} \equiv\left\{V_{1} / D_{p 1}+V_{2} / D_{p 2}-(2 / 3) \pi R_{1}^{3} I^{\prime} A\right\} /\left(\pi R_{i}^{2}\right) \\
& D_{i} \equiv \Gamma A R_{1}^{3} / R_{i}^{2}, \quad \Gamma \equiv 1 / D_{p 2}-1 / D_{p 1} \\
& D_{p j} \equiv d_{p j} \cdot \varepsilon_{j} /\left(1-\varepsilon_{j}\right), \quad(j=1,2) \quad \ldots \ldots \ldots . . .
\end{aligned}
$$

Substituting Eq. (5) into Eq. (1), we have

$$
\partial P / \partial l=\left\{\rho \Psi /\left(g_{c} \phi \Delta_{i}\right)\right\}\left(C_{i}+D_{i} \zeta\right) \cdot u(\zeta)^{2}
$$

If the pressure gradient expressed by Eq. (7) is approximately same at any radial position, Eq. (8) can be obtained.

$$
\partial(\partial P / \partial l) / \partial \zeta=0
$$

On the other hand, the mass balance regarding gas gives Eq. (9);

$$
F=2 \pi R_{i}^{2} \int_{0}^{1} \zeta \cdot \bar{\varepsilon}(\zeta) \cdot u(\zeta) d \zeta
$$

Combination of Eqs. (7) through Eq. (9) yields the expression for the radial velocity profiles as:

$$
u_{N}=\underset{2 \pi R_{i}^{2} E_{i}}{F} \cdot \frac{A+B \zeta}{\sqrt{ } C_{i}+D_{i} \zeta}
$$

where $E_{i}$ is a dimensionless factor defined as:

$$
\begin{aligned}
E_{i} \equiv & 2\left[\sqrt{ } C_{i}+D_{i}\left\{B\left(C_{i}+D_{i}\right)^{2} / 5+\left(D_{i} A-2 B C_{i}\right)\right.\right. \\
& \left.\cdot\left(C_{i}+D_{i}\right) / 3-C_{i}\left(D_{i} A-B C_{i}\right)\right\}-\sqrt{ } C_{i}\left\{B C_{i}^{2} / 5\right. \\
& \left.\left.+C_{i}\left(D_{i} A-2 B C_{i}\right) / 3-C_{i}\left(D_{i} A-B C_{i}\right)\right\}\right] / D_{i}^{3} \ldots \ldots
\end{aligned}
$$

Equation (10) will give the resultant radial distribution of the flow rate of gas, when the changes in the apparent angles of repose, o/c and coke base are caused due to the different charging method. Theoretical analysis developed in this paper can be applied also to beds having other contours, e.g., M-shaped contour. 


\section{Mathematical Model of Blast Furnace with Radially Distributed Burdens}

Because the apparent angles of repose of the ore and the coke burdens differ to each other, the vertical depth of each layers varies radially. Consequently, the radial distributions would be expected concerning not only the flow rate of gas but also the longitudinally averaged properties such as o/c, specific heat of solid particles, heat transfer coefficient between gas and solid particles, and specific surface area.

The modeling procedures proposed in the previous mathematical model ${ }^{16)}$ was applied to the region from $\zeta$ to $(\zeta+d \zeta)$ where both the gas and the solid particles are assumed to be in a piston flow. Then, the equations have been obtained to express the unsteady-state heat transfer, the overall mass balance and the pressure drop of the gas in the ore and coke burdens within $i$-th block. The present equations are similar to the equations presented in the previous paper, ${ }^{16}$ i.e., Eqs. (6) to (9), Eqs. (17) to (27) and Eqs. (28) to (31), respectively. However, the flow rate of gas through the region has to be estimated from Eq. (10) of this paper.

In deriving the overall heat balance equation for each layer, the heat transferred from the $m$-th cell to the radial direction during time period of $\Theta, Q_{i, m}$, was taken into account. It has been expressed by:

$$
\begin{aligned}
Q_{i, m} & =2 \pi R_{i, m} \cdot \Delta_{j, i} \cdot\left(\xi_{m}^{\prime} U\right) \int_{0}^{\Theta}\left(T_{m}-T_{w}\right) d \theta \\
& -2 \pi R_{i, m-1} \cdot \Delta_{j, i} \cdot\left(\xi_{m-1}^{\prime} U\right) \int_{0}^{\Theta}\left(T_{m-1}-T_{w}\right) d
\end{aligned}
$$

For the sake of further simplification, $Q_{i, m}$ has been written as follows:

$$
\left.Q_{i, m}=2 \pi R_{i, m} \cdot\right\lrcorner_{j, i} \cdot(\xi U) \int_{0}^{\Theta}\left(T_{m}-t_{m}\right) d \theta
$$

Upon using Eq. (13) and integrating the unsteadystate heat transfer equations for each layer (Eqs. (6) and (8) of the previous paper $\left.{ }^{\mathbf{1 6}}\right)$ over the range of $\theta=0 \sim \Theta$, the temperature of gas flowing into the ore and the coke layers can be expressed by Eqs. (14) and (15), respectively.

$$
\begin{aligned}
& T_{1, i}=L_{2, i} \cdot T_{2, i} \\
& \left.\left.+\gamma_{2, i}(\lrcorner_{2, i} /\right\lrcorner_{i}\right)\left(t_{2, i}-M_{2, i} \cdot t_{2, i-1}-J T_{\mathrm{o}, i}-J T_{1, i}\right) \\
& \left.+\mathcal{N}_{2, i} / T_{2, i}-t_{w}\right) \\
& T_{2, i+1}=L_{1, i} \cdot T_{1, i} \\
& \left.+\gamma_{1, i}\left(\Delta_{1, i} /\right\lrcorner_{i}\right)\left(t_{1, i}-M_{1, i} \cdot t_{1, i-1}-J T_{c, i}\right) \\
& +\mathcal{N}_{1, i}\left(T_{1, i}-t_{w}\right)
\end{aligned}
$$

where the factors which were similarly defined elsewhere $^{16)}$ can be written as:

$$
\begin{aligned}
L_{1, i} & \equiv F_{1, i} \rho_{1, i} c_{1, i} / F_{2, i+1} \rho_{2, i+1} c_{2, i+1} \\
L_{2, i} & \equiv F_{2, i} \rho_{2, i} c_{2, i} / F_{1, i} \rho_{1, i} c_{1, i} \\
M_{j, i} & \equiv \rho_{b j, i-1} c_{s j, i-1} / \rho_{b j, i} c_{s j, i} \quad(j=1,2) \\
\gamma_{1, i} & \equiv F_{s} \rho_{b 1, i} c_{s 1, i} / F_{2, i+1} \rho_{2, i+1} c_{2, i+1} \\
\gamma_{2, i} & \equiv F_{s} \rho_{b 2, i} c_{s 2, i} / F_{1, i} \rho_{1, i} c_{1, i}
\end{aligned}
$$

$$
\begin{aligned}
& J T_{c, i} \equiv C_{c, 0}\left(f_{c, i}-f_{c, i-1}\right)\left(-\Delta H_{c, i}\right) / \rho_{b 1, i} c_{s 1, i} \\
& J T_{k, i} \equiv C_{k, 0}\left(f_{k, i}-f_{k, i-1}\right)\left(-\Delta H_{k, i}\right) / \rho_{b 2, i} c_{s 2, i} \\
& (k=\mathrm{o}, l)
\end{aligned}
$$

while $\mathcal{N}_{j, i}$ denoting the dimensionless factor concerning the radial heat transport is recast as $\mathbf{E q}$. (17). In the present study, furthermore, it is also to be noted that the vertical depth of each layer, $\Delta_{j, i}$, varies in the radial as well as vertical direction according to Eq. (17).

$$
\begin{aligned}
\mathcal{N}_{1, i} & \equiv 2 \pi \zeta R_{i} \Delta_{1, i} \xi U / F_{2, i+1} \rho_{2, i+1} c_{2, i+1} \\
\mathcal{N}_{2, i} & \left.\equiv 2 \pi \zeta R_{i}\right\lrcorner_{2, i} \xi U / F_{1, i} \rho_{1, i} c_{1, i} \\
J_{1, i} & \equiv\left\{V_{1}+\pi R_{1}^{3} \Lambda(2 / 3-\zeta)\right\} / \pi R_{i}^{2} \\
J_{2, i} & \equiv\left\{V_{2}-\pi R_{1}^{3} \Lambda(2 / 3-\zeta)\right\} / \pi R_{i}^{2} \\
J_{i} & =J_{1, i}+\Delta_{2, i} \ldots \ldots \ldots \ldots \ldots \ldots \ldots \ldots \ldots \ldots \ldots \ldots \ldots \ldots \ldots
\end{aligned}
$$

The numerical solutions of the previously reported equations (concerning the unsteady-state heat transfer, the overall mass balance and the pressure drop) together with the overall heat balance equations (shown above (Eqs. (14) and (15)), will give the longitudinal and the radial distributions of the process variables in the furnace. The value of the overall heat transfer coefficient, $U$, used in this calculation was $15 \mathrm{kcal} / \mathrm{m}^{2} \cdot \mathrm{hr} \cdot{ }^{\circ} \mathrm{C}$ determined from the previous estimations. ${ }^{16,23)}$ To obtain the value of $\xi$, the effective thermal conductivity ${ }^{24)}$ has been applied for analyzing the observed results ${ }^{25}$ ) regarding the temperature distribution in cylindrical bed. As the result, the following simple correlation has been obtained.

$$
\xi=\zeta
$$

The testing calculations has been carried out for two cases where the furnace was radially divided into 5 and 10 sections. The calculated temperature profiles between both cases were nearly same. This may suggest that the sensitivity of the term $(\xi U)$ in the model may be fairly small. Thus, the sensible heat transfer in the vertical direction and the heat generation by the chemical reactions would play an important role on the thermal states in the furnace, but the sensible heat transfer in the radial direction would not. Consequently, it is good to formulate the radial heat transport simply by Eqs. (13) and (18).

\section{Numerical Analysis of Distributions of Process Variables}

The numerical analysis of the inner features of B.F. (DII) has been performed by applying the same operating conditions as used in the previous paper. ${ }^{16)}$

\section{Determination of Initial Conditions}

The initial conditions at the top of the bed are given by the radial distributions concerning the burdens, the flow rate of gas, the gas temperature and the gas compositions.

When the values are given for the volumes of each layer $\left(V_{j}\right)$, the apparent angle of repose $\left(\alpha_{j}\right)$ and the furnace diameter at the top of bed $\left(R_{1}\right)$, the radial distribution of the burdens can be evaluated from Eq. 
(17) as the radial change in the thickness of each layer, $\Delta_{j}$. Furthermore, after the volume flow rate of gas flowing through the shaft is determined from the overall mass balances over the whole furnace, Eq. (10) gives its radial distribution under the given charging conditions.

Figure 2 shows the calculated radial profiles of $\bar{\varepsilon}$, $\bar{d}_{p}$ and $u_{N}$ in the first block at the top of B.F. (DII) having $\alpha_{1}=25^{\circ}$ and $\alpha_{2}=28^{\circ}$. It can be found from Fig. 2 that the respective ratios of the values on the center, axis to those on the peripheral wall amount to 1.07 for $\bar{\varepsilon}, 1.22$ for $\bar{d}_{p}$ and 1.26 for $u_{N}$. Accordingly, the ascending gas tends to flow through the central zone more than the peripheral zone in the furnace.

As for the distributions of the temperature and the compositions of the top gas in an operating furnace, it may be difficult to measure the time-averaged values of their radial profiles during a charging period of each burdens $(\theta=0 \sim \Theta)$. However, their values averaged with respect to the total flow rate of gas can be measured without difficulty as is often the case with the industrial works.

Thus, the following procedures have been used to determine their radial profiles at the top of bed. First, let their mean values averaged with respect to the total flow rate of gas be represented by Eq. (19) during the period of $\Theta$. Second, to the term of $\lambda_{0}(\zeta)$ in Eq. (19) their typical radial profiles measured at any moment in the blast furnace (in this case, B.F.(DII)) are applied. Third, if the value of $\bar{\lambda}_{0}$ calculated from Eq. (19) differs to some extent from the observed, the successive modifications of the pattern of $\lambda_{0}(\zeta)$ are repeated until the final pattern is obtained.

$$
\bar{\lambda}_{0}=\left\{2 \pi R_{1}^{2} \int_{0}^{1} \zeta \cdot u_{N}(\zeta) \cdot \lambda_{0}(\zeta) d \zeta\right\} / F
$$

where, $\quad \lambda_{0}=T_{0}, x_{0}, y_{0}, v_{0}, w_{0}$.

However, the radial change in $v_{0}$ and $w_{0}$ were neglected in the numerical analysis of B.F. (DII). Figure 3 shows the radial profiles of the temperature and the compositions of $\mathrm{CO}$ and $\mathrm{CO}_{2}$ of the top gas in B.F. (DII), determined by the procedures mentioned above.

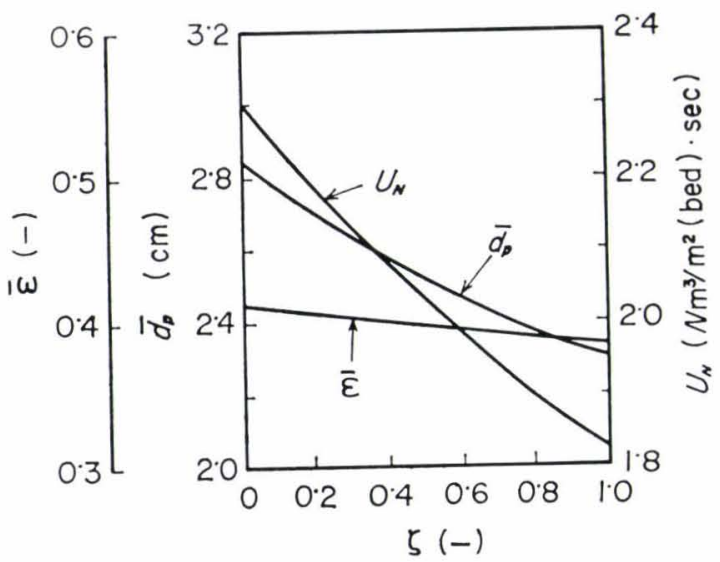

Fig. 2. Radial distributions of $\bar{\varepsilon}, \bar{d}_{p}$ and $u_{N}$ at the top of B.F. (DII)

\section{Results of Numerical Analysis}

The longitudinal variations in the process variables along each radial position have been numerically analyzed, based on the mathematical model mentioned above and the initial conditions given at the top of bed. Considering the melting positions of the ore burdens to be equal to the locations of the isotherm of $1400^{\circ} \mathrm{C}$, the computations were brought to an end at the positions. When the stock column of B.F. (DII) was radially divided into 5 sections, the computation time required was about $70 \mathrm{sec}$ on FACOM 230-60 digital computer at Nagoya University Computation Center.

\section{Longitudinal Distribution of Temperature}

It has been found that both the temperatures of the gas and the solid particles calculated at each radial position oscillate in the vertical direction similarly as reported in the previous paper, ${ }^{\mathbf{1 6})}$ and the amplitudes of these oscillations tend to enlarge in the central region.

Figure 4 shows the radial distribution of the timeaveraged temperature of the solid particles. It may be found from Fig. 4 that at any radial position except the central region, two steps of thermal reserve zones are distinctly formed in the upper and the lower zones of the furnace. The temperature of the upper reserve zone $\left(t_{R 1}\right)$ greatly changes in the radial direction and $t_{R 1}$ in the central region is higher than the peripheral region (i.e., $t_{R 1}=850^{\circ} \mathrm{C}$ at $\zeta=0.3$ and $t_{R 1}=600^{\circ} \mathrm{C}$ at $\zeta=0.9)$. The vertical depth of the upper reserve zone becomes long toward the peripheral direction.

As for the lower reserve zone, on the other hand, the radial change in its temperature $\left(t_{R 2}\right)$ is comparatively slight and $t_{R 2}$ is kept within the temperature range of 1000 to $1100^{\circ} \mathrm{C}$, where the Boudouard reaction becomes active. Consequently, it may be considered that $t_{R 2}$ depends strongly on the reactivity of the cokes.

By the use of vertical probes, Okabe, et al. ${ }^{6)}$ measured the longitudinal distribution of the temperature in the shaft of an operating furnace, and they found the existence of a thermal reserve zone having the temperature $\left(700^{\circ}\right.$ to $800^{\circ} \mathrm{C}$ ) lower than that observed previously. As is expected from their method for

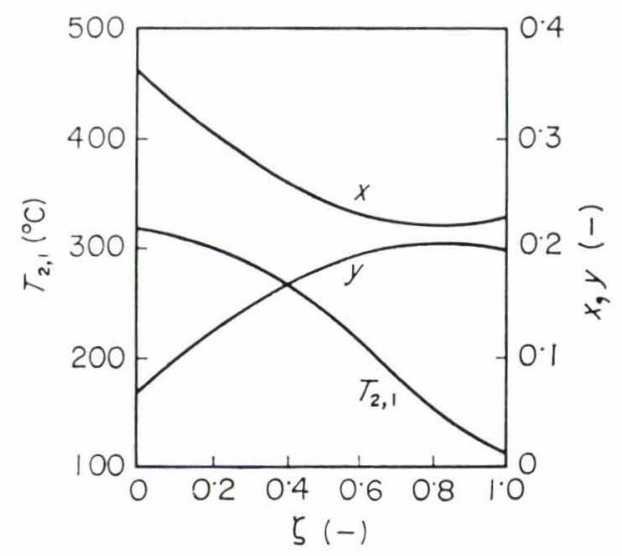

Fig. 3. Radial distributions of $x, y$ and $T_{2,1}$ at the top of B.F. (DII) 


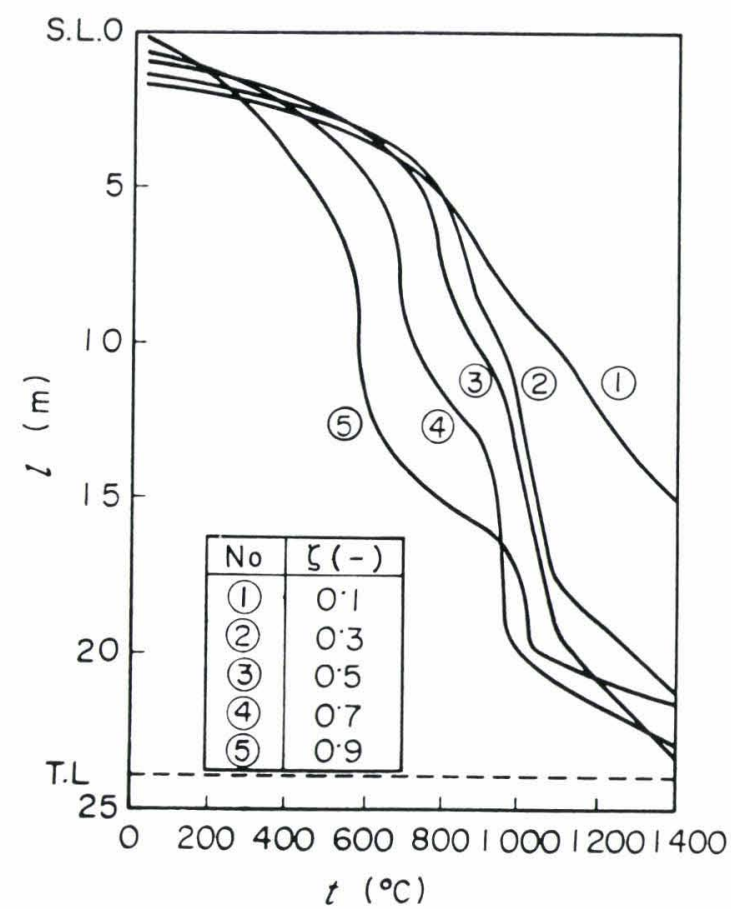

Fig. 4. Longitudinal distributions of temperature of solid particles in B.F. (DII)

measurement, it may be presumed at their observed data corresponds to $t_{R 1}$ in the periph, ral region.

When the burdens are heated to tı e higher temperature than $t_{R 2}$, the endothermic reaction of Boudouard reaction becomes still more active. Consequently, the temperatures of the gas and the solid particles change largely in the lower part of the furnace. Especially in the peripheral region having the large value of $\mathrm{o} / \mathrm{c}, \mathrm{CO}_{2}$ is violently generated by the indirect reduction, and the temperatures remarkablly changes.

\section{Radical Distribution of Temperature}

The isotherms of the solid particles from the top to the melting level are shown in Fig. 5 at intervals of $100^{\circ} \mathrm{C}$. In the neighborhood of the top of the bed, the solid temperature is higher in the peripheral region than in the central region. These result depend on the top contour of burdens.

In the lower region of 4 to $5 \mathrm{~m}$ below the top, the solid temperature is oppositely higher in the central region than in the peripheral region. This may be caused by the combined effects of the various thermal conditions as mentioned below. Namely, in the central region, the value of $h_{p}$ becomes large owing to the large flow rate of gas in that region. According to the large flow rate and the high temperature of gas, the heat transfer becomes rapid from gas to solid particles. On account of the radially distributed o/c and the gas flow the longitudinally averaged thermal flow ratio $(\bar{\gamma})$ as defined by the following equation (20) is large in the peripheral region $(\bar{\gamma}=0.87)$ and small in the central region $(\bar{\gamma}=0.72)$. Near the periphery, the large value of $\mathrm{o} / \mathrm{c}$ causes the large amount of heat removal due to the indirect reduction $\left(\mathrm{Fe}_{3} \mathrm{O}_{4} \rightarrow \mathrm{FeO}\right)$.

$$
\bar{\gamma} \equiv\left(\gamma_{1} V_{1}+\gamma_{2} V_{2}\right) /\left(V_{1}+V_{2}\right)
$$

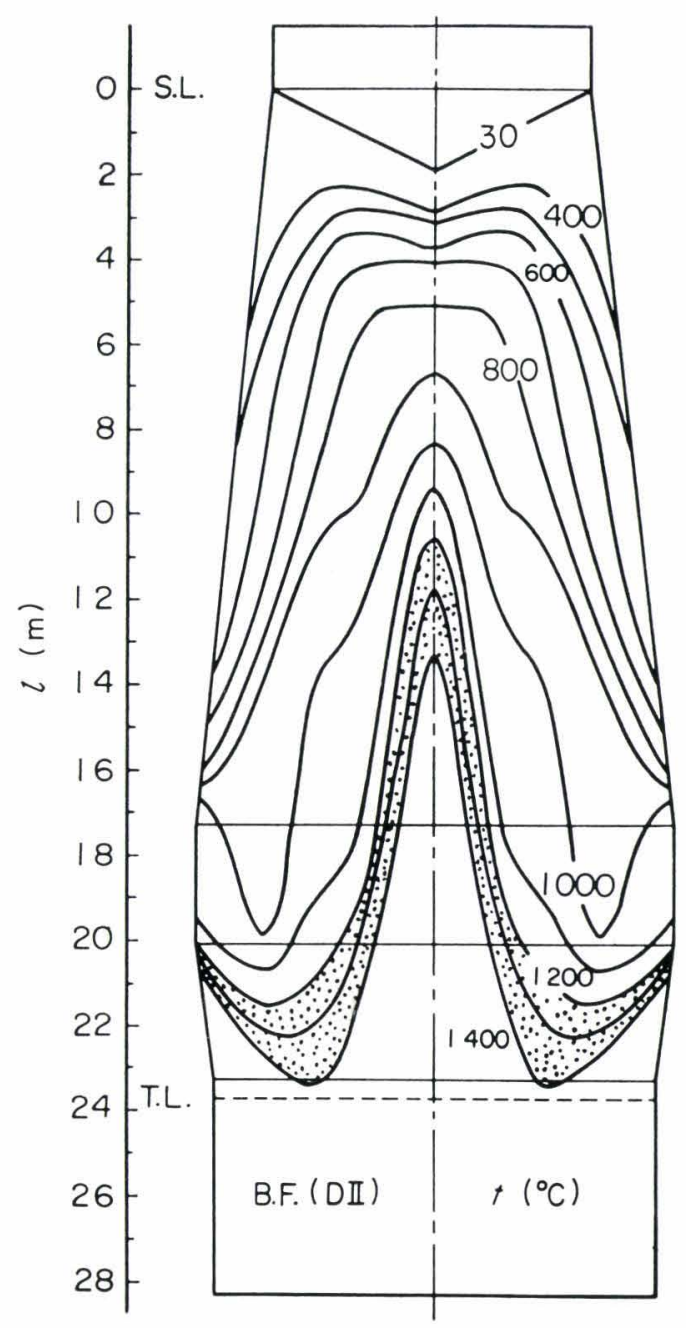

Fig. 5. Distributions of isotherms in B.F. (DII)

Although the temperature profiles in the furnace may differ according to the differences in the operating conditions, it may be seen from Fig. 5 that the calculated patterns of the isotherms are fairly similar to the results observed by Kinney ${ }^{1)}$ in the shaft by the use of the probes.

\section{Locations of Melting Down and Deadman}

Assuming the melting temperature of the ore particles as $1400^{\circ} \mathrm{C}$ approximately, the locations of melting down may be given by the isotherm of $1400^{\circ} \mathrm{C}$. Figure 5 illustrates the calculated patterns of isotherms.

It is considered that the solid particles below the isotherm of $1400^{\circ} \mathrm{C}$ may be consisted of coke alone. Then if we assume the coke zone having the conical shape as the deadman, Fig. 5 would suggest the existence of the deadman whose angle of repose amounts to about $80^{\circ}$.

Recently, Kanbara, et al. ${ }^{13)}$ observed the inner features of the practical blast furnace quenched by nitrogen gas. A number of annular layers, in which the iron ores had been softened and partly molten, were detected over the zone from the tuyere to the middle level of the shaft. Regarding Fig. 5 such softening layers as mentioned above may be considered to distribute in the range surrounded by the 
isotherms of $1200^{\circ}$ and $1400^{\circ} \mathrm{C}$. It is noted that the pattern of this region illustrated in Fig. 5 is similar to the findings ${ }^{13}$ in the dissected furnace.

4. Distributions of Compositions of $\mathrm{CO}$ and $\mathrm{CO}_{2}$

Figure 6 shows the longitudinal distributions of $x$ and $y$ at each given radial position. The values of $x$ and $y$ oscillate at each radial position, and their amplitudes are illustrated by the two curves, respectively. The radial distributions of $x$ and $y$ become predominant particularly in the shaft.

As is obvious in Fig. 6, the value of $x$ reaches to the maximum (about 0.4) to form a peak at every radial position in the temperature range where the Boudouard reaction becomes predominant. In the zone above the peak the amplitude of $x$ is larger in the central side than in the peripheral side, but the reverse tendency appears in the zone below the peak.

On the other hand, the value of $(x+y)$ remains constant at about $42 \%$ in the zone above the peak, while it decreases progressively with the increase in the distance down the peak. Ultimately in the melting level, it amounts to about $39 \%$ at every radial position.

The gas utilization of $\mathrm{CO}$ is generally defined as, $\eta=100 \cdot y /(x+y) \%$. When the solid temperature is less than $600^{\circ}$ to $800^{\circ} \mathrm{C}$, the value of $\eta$ becomes nearly constant (this value: $\eta_{s}$ ). When the Boudouard reaction proceeds rapidly at the temperature above $1000^{\circ}$ to $1100^{\circ} \mathrm{C}, \eta$ decreases rapidly and reaches to about 1 to $3 \%$ at every radial position. Moreover, it has been detected that the variations of $\eta$ at the intermediate temperature range $\left(800^{\circ}\right.$ to $\left.1000^{\circ} \mathrm{C}\right)$ can be plotted in the neighborhood of the equilibrium curve of $\mathrm{FeO}-\mathrm{Fe}$. The tendencies of the variations of these calculated results have almost coincided with the results observed by using the probe ${ }^{6,21)}$ in the vertical zone near the furnace wall. However, the values of $\eta_{s}$ were largely different in the radial direction. Namely, the values of $\eta_{s}$ of about 23, 34, 43, 48 and $48 \%$ were obtained at each $\zeta$ being $0.1,0.3$, $0.5,0.7$ and 0.9 , respectively.

\section{Distributions of Gas Velocity}

Figure 7 shows the longitudinal distributions of the gas velocity at each given radial position. Where, $u_{1}$ and $u_{2}$ denoted the velocities in the coke layer and the ore layer, respectively. It may be seen from Fig. 7 that every velocity at each given 5 reaches to

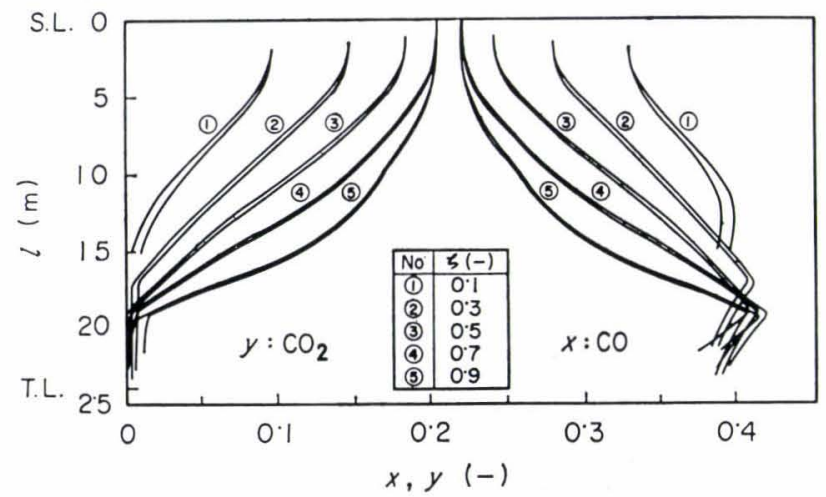

Fig. 6. Longitudinal distributions of $x$ and $y$ in B.F. (DII) the highest value in the neighborhood of $l=3$ to $4 \mathrm{~m}$.

As for the radial distributions of the gas velocity, Fig. 7 shows that the velocities in the central region are higher than those in the peripheral region, and that the distributions become remarkable near the top zone.

\section{Distributions of Gas Pressure}

Figure 8 shows the longitudinal distributions of the gas pressure $(P)$ at each given radial position. Figure 8 may suggest that $P$ in the peripheral region near the top becomes higher than $P$ in the central region owing to the effect of the profiles of the top contour of the burdens.

Polthier ${ }^{18)}$ measured the pressure of gas flowing through the isothermal packed bed (the inner diameter: $D$ ) having $\mathrm{V}$-shaped top contour, and found that the top contour exerted the influence on $P$ up to

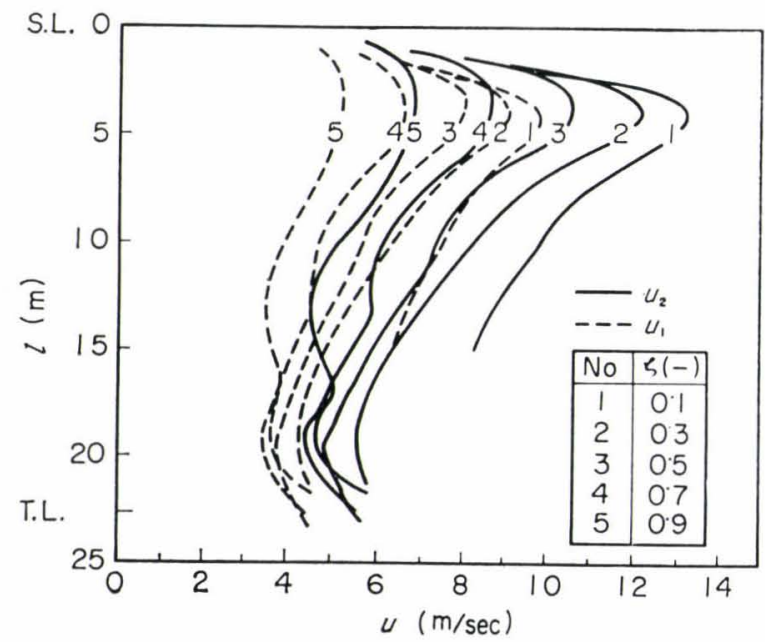

Fig. 7. Longitudinal distributions of gas velocities in B.F. (DII)

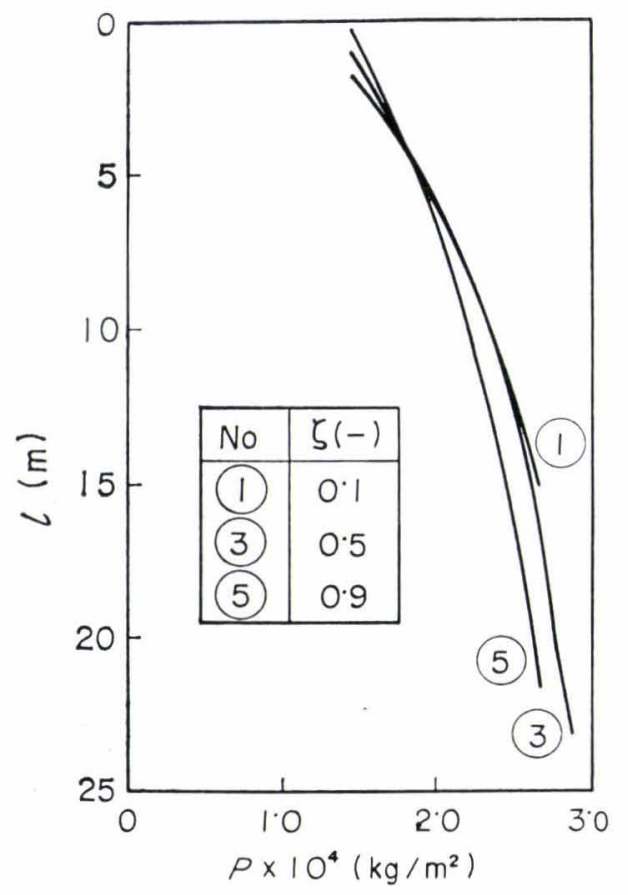

Fig. 8. Longitudinal distributions of gas pressure in B.F. (DII) 
the level below by $0.5 \mathrm{D}$ from the presumably evened top level.

For the case of B.F. (DII), the equivalent length to $0.5 \mathrm{D}$ amounts to about $4 \mathrm{~m}$ in the top zone. Now, it is found from Fig. 8 that the gas pressures at each given $\zeta$ become equal to each other at the level below by 4 to $5 \mathrm{~m}$ from stock line. In the zone below more than $5 \mathrm{~m}$ from stock line, however, $P$ in the central region becomes higher than in the peripheral region in contrast with the top zone. Moreover, it is noted that the pressure gradients in the longitudinal direction of every curve have a tendency to come progressively to the constant value with the increased distance from stock line. Consequently, the insignificant change in the patterns of the radial distributions of $P$ may be detected in the longitudinal direction. Such results mentioned above are similar to the results measured by Kinney ${ }^{1)}$ in an operating blast furnace.

\section{Distributions of Fractional Reduction}

Figure 9 shows the longitudinal distributions of $f_{0}$ at each radial position. Fuigre 10 shows the distributions of the equi-fractional reduction of iron ore. In these figures, the dotted lines represent the isotherm of $1400^{\circ} \mathrm{C}$ assumed as the melting level. As mentioned previously, the numerical computations were brought to an end at the location of this isotherm.

From Fig. 9 we may find the following characteristics: At every radial position the reduction speed is slow in the upper part of the furnace, while it is rapidly accelerated in the middle part. However, it gets to decrease again when high value of $f_{\mathrm{O}}$ is attained. Furthermore, the value of $f_{0}$ in the central region is higher than that in the peripheral region. Figure 10 otherwise shows the similar results to those pictured in Fig. 9.

Byalyi and $\mathrm{Kotov}^{4}$ ) measured the radial distributions of $f_{0}$ at three levels of an operating blast furnace by inserting a probe into the shaft. From these ob-

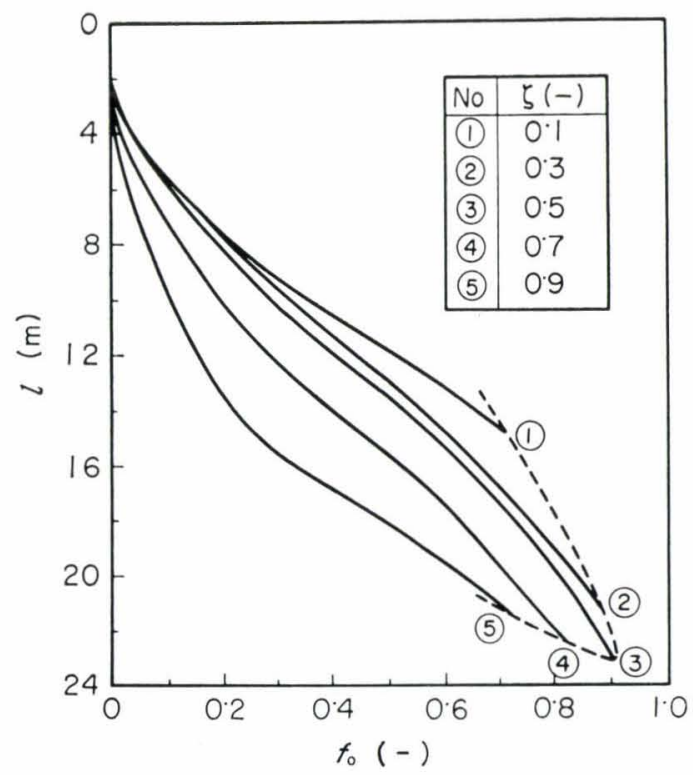

Fig. 9. Longitudinal distributions of fractional conversion in B.F. (DII) served results, they reported the large differences in the values of $f_{0}$ between the central region and the peripheral region. The former at the middle level and the lower level of the shaft reached to 45 and $80 \%$, respectively. On the other hand, the latter at the corresponding levels amounted only to 15 and $20 \%$, respectively. Their observations are fairly in good agreement with the calculated results shown in Fig. 10 .

Moreover, Fig. 9 shows that the values of $f_{0}$ at $1400^{\circ} \mathrm{C}$ amount to about $71,88,90,82$ and $73 \%$ at each position of $\zeta=0.1,0.3,0.5,0.7$ and 0.9 , respectively. That is, in the intermediate region of the furnace the indirect reduction yields the high value of $f_{0}$. Thus the contribution of the direct reduction of molten $\mathrm{FeO}$ will become to the small extent.

\section{Conclusions}

A mathematical model has been proposed on the radial distributions of process variables over the region from the top to the melting level of the furnace with the inclined layered burdens. The formulation is developed by extending the previously reported ${ }^{16)}$ mathematical model of the blast furnace with the horizontally layered burdens.

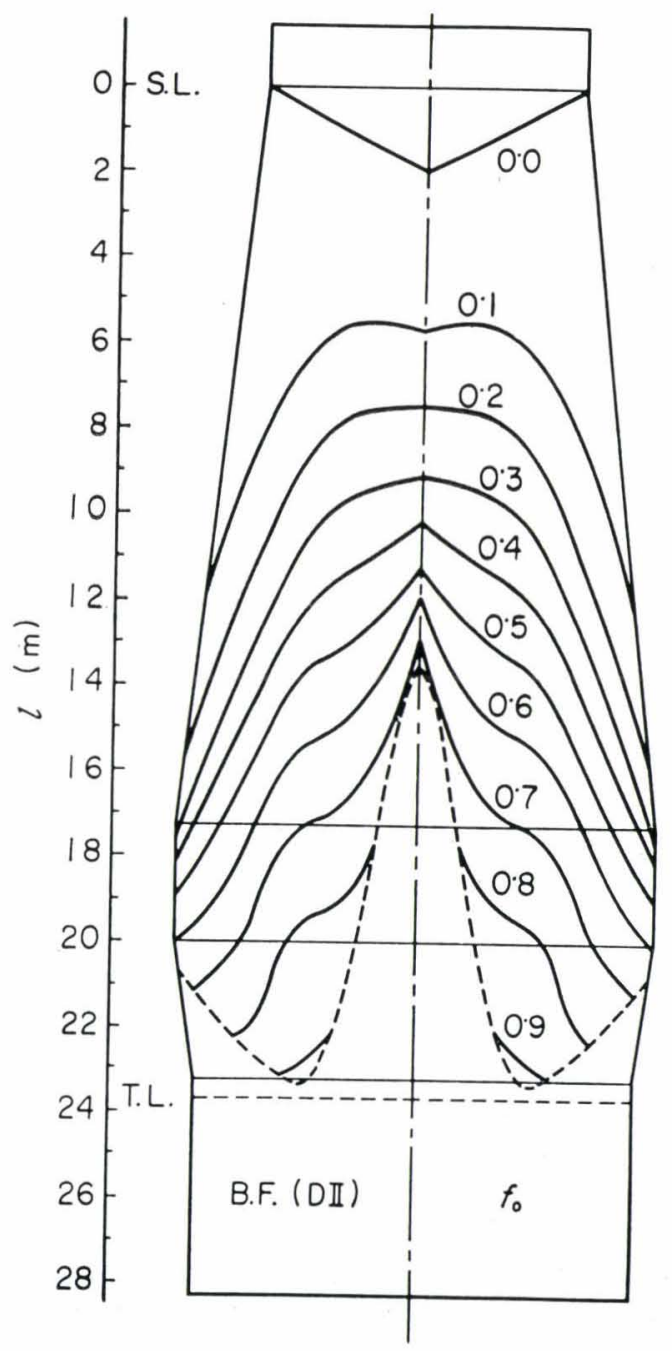

Fig. 10. Distributions of equi-fractional reduction in B.F. (DII) 
By using the model, the inner features of a blast furnace (B.F. (DII)) operating in Japan were calculated with the aid of a digital computer. The computed results show that the process variables (such as the temperature, the gas composition, the gas velocity and the fractional reduction of iron ore) and the softening layer of iron ores (over the region from $1200^{\circ}$ to $1400^{\circ} \mathrm{C}$ ) change remarkably in the radial direction. It may be considered that the predicted inner features of the furnace are similar to the results observed by the other investigators (e.g., those measured by the probe and those from the dissected furnace).

According to the model proposed in this paper, the distributed process variables in the operating blast furnace can be estimated on the basis of the operating conditions and the measurable data at the furnace top. Because the necessary time for these calculations is considerably short, this model can be easily used for determining the reasonable operating conditions.

\section{Nomenclature}

$A, B:$ factors defined by Eq. (4) (-)

$C, D:$ factors defined by Eq. (6) (-)

$c$ : specific heat of gas $\left(\mathrm{kcal} / \mathrm{kg} \cdot{ }^{\circ} \mathrm{C}\right.$ )

$c_{s j}$ : specific heat of solid particles averaged by taking account of composition in $j$-th layer $(j=1,2)\left(\mathrm{kcal} / \mathrm{kg} \cdot{ }^{\circ} \mathrm{C}\right)$

$d_{p j}: \quad$ particle diameter in $j$-th layer $(j=1,2)(\mathrm{m})$

$\bar{d}_{p}$ : mean particle diameter of adjacent two layers of coke and ore (m)

$E$ : factor defined by Eq. (11) (-)

$F$ : volume flow rate of gas $\left(\mathrm{Nm}^{3} / \mathrm{hr}\right)$

$F_{s}$ : volume flow rate of solid particles $\left(\mathrm{m}^{3}\right.$ (one block)/hr)

$f_{\mathrm{o}}$ : fractional conversion of iron ore $(-)$

$g_{e}:$ conversion factor $\left(\mathrm{kg} \cdot \mathrm{m} / \mathrm{Kg} \cdot \mathrm{hr}^{2}\right)$

$h_{p}$ : heat transfer coefficient between gas and solid particle $\left(\mathrm{kcal} / \mathrm{m}^{2} \cdot \mathrm{hr} \cdot{ }^{\circ} \mathrm{C}\right)$

$L_{j}, M_{j}, \mathcal{N}_{j}$ : factors defined by Eqs. (16) and (17) (-)

$l$ : distance from stockline $(\mathrm{m})$

$P$ : pressure of gas $\left(\mathrm{Kg} / \mathrm{m}^{2}\right)$

$Q:$ heat transferred radially during time period $\Theta$ (kcal)

$R_{i}$ : mean radius of $i$-th block in blast furnace (m)

$R e_{p}$ : Reynolds' number defined by Eq. (2) (-)

$r$ : radius of blast furnace $(\mathrm{m})$

$T$ : temperature of gas $\left({ }^{\circ} \mathrm{C}\right)$

$\Delta T_{c}, \Delta T_{l}, \Delta T_{\mathrm{O}}$ : factors defined by Eq. (16) ( $\left.{ }^{\circ} \mathrm{C}\right)$

$t_{j}$ : temperature of solid particles in $j$-th layer $\left({ }^{\circ} \mathrm{C}\right)$

$t_{R}$ : temperature of thermal reserve zone in blast furnace $\left({ }^{\circ} \mathrm{C}\right)$

$t_{w}$ : temperature of cooling water $\left({ }^{\circ} \mathrm{C}\right)$

$U$ : overall heat transfer coefficient through furnace wall based on inner surface area $\left(\mathrm{kcal} / \mathrm{m}^{2} \cdot \mathrm{hr} \cdot{ }^{\circ} \mathrm{C}\right)$

$u$ : linear velocity of gas $(\mathrm{m} / \mathrm{hr})$

$u_{N}$ : superficial flow rate of gas $\left(\mathrm{Nm}^{3} / \mathrm{m}^{2}\right.$ (bed). $\mathrm{hr}$ )

$u_{1}, u_{2}$ : gas velocity in coke layer and ore layer, respectively $(\mathrm{m} / \mathrm{hr})$
$V_{j}: \quad$ volume of $j$-th layer per one charge $\left(\mathrm{m}^{3}\right.$ (bed)) $v, w, x, y$ : molar fraction of water vapor, $\mathrm{H}_{2}, \mathrm{CO}$, $\mathrm{CO}_{2}$, respectively (-)

$\alpha_{j}$ : apparent angle of repose of $j$-th layer $(j=1,2)$ $(-)$

$\Gamma: \quad$ factor defined by Eq. (6) $(1 / \mathrm{m})$

$\gamma_{j}$ : thermal flow ratio in $j$-th layer (defined by Eq. (16)) (-)

$\bar{\gamma}$ : average thermal flow ratio of adjacent two layers of coke and ore (defined by Eq. (20)) $(-)$

$\Delta_{i}: \quad$ vertical depth of $i$-th block (m)

$\Delta_{j, i}$ : vertical depth of $j$-th layer in $i$-th block (m)

$\varepsilon_{j}: \quad$ voidage in $j$-th layer $(j=1,2)(-)$

$\bar{\varepsilon}$ : mean voidage of adjacent two layers of coke and ore (-)

$\zeta$ : dimensionless radius in furnace $\left(=r / R_{i}\right)$ $(-)$

$\eta$ : gas utilization of $\mathrm{CO} \quad(\%)$

$\Theta$ : residence time of solid particles in each block (hr)

$\theta$ : time (hr)

A: factor defined by Eq. (4) (-)

$\nu$ : kinematic viscosity of gas $\left(\mathrm{m}^{2} / \mathrm{hr}\right)$

$\xi, \xi^{\prime}$ : correction factor of $U$ defined by Eq. (13) and Eq. (12), respectively (-)

$\rho:$ density of gas $\left(\mathrm{kg} / \mathrm{Nm}^{3}\right)$

$\rho_{b j}: \quad$ bulk density of $j$-th layer $\left(\mathrm{kg} / \mathrm{m}^{3}(j\right.$-th layer $\left.)\right)$

$\phi: \quad$ shape factor of solid particles (-)

$\bar{\phi}$ : mean $\phi$ of adjacent two layers of coke and ore (-)

$\Psi:$ drag coefficient in Ergun's equation (-)

$\bar{T}$ : mean $\Psi$ of adjacent two layers of coke and ore (defined by Eq. (2)) (-)

\section{Subscripts}

0: top of furnace

$i$ : block number marked from top level to tuyere level of furnace

$j$ : layer number $(j=1,2 ; 1$ : coke layer, 2 : ore layer)

$m$ : cell number marked from center line to radial direction

\section{REFERENCES}

1) S. P. Kinney: Techn. Paper Bur. Mines, Nr. 442 (1929); ref. Stahl u. Eisen, 49 (1929), 1728.

2) B. L. Lazarev, B. I. Kitaev and Yu. G. Yaroshenko: Stal in Eng., (1961), 162.

3) B. L. Lazarev, B. A. Bokoviskov, N. M. Babushkin, V. N. Timofeev, V. V. Chervotkin and S. I. Privalov: Stal in Eng., (1965), 429.

4) L. A. Byalyi and A. P. Kotov: Stal in Eng., (1966), 956.

5) S. Fujii, S. Tamura, K. Taguchi, K. Kunii and R. Nishida: Tetsu-to-Hagané, 54 (1968), 1241.

6) K. Okabe, T. Hamada and A. Watanabe: Tetsu-to-Hagané, 55 (1969), 764.

7) Z. I. Nekrasov, V. L. Pokryshkin, P. G. Netrebko, A. A. Zaloga, M. T. Buzoverya, V. T. Os'kin, E. Ya Aksenich, I. Ya. Brainin, V. E. Gerashchenko, B. F. Marderand and V. I. Bondarenko: Stal in Eng., (1970), 502.

8) B. N. Starshinov, Yu. A. Orlov, A. G. Khoruzhi, Yu. V. Vyazovskii, S. V. Vasil'ev, G. G. Lukashov and G. B. Ravi- 
novich: Stal in Eng., (1970), 849.

9) J. J. Bosley, B. Melcher and M. M. Harris: J. Metals, 11 (1959), 610 .

10) K. Kodama and S. Hashimoto: Proceedings of ICSTIS, I, ISIJ, (1971), 112.

11) R. Ando, T. Miyashita, T. Shimotsuma, T. Kaburagi and Y. Yamada: Nippon Kokan Techn. Rep., 54 (1971), 471.

12) M. Tate: Report of the 54th Committee of Japan Soc. for the Promotion of Science, No. 1227, (1972).

13) K. Kanbara, T. Hagihara, M. Sasaki, Y. Okuno, T. Katayama and K. Yoshizawa: Tetsu-to-Hagané, 59 (1973), A77.

14) Y. Kajikawa, N. Sumida, H. Yoshikoshi, T. Fukushima, T. Kamoshida and T. Fukuyama: Tetsu-to-Hagané, 59 (1973), A81.

15) A. K. Lahiri and V. Seshadri: JISI, 207 (1969), 293.

16) M. Kuwabara and I. Muchi: Tetsu-to-Hagané, 61 (1975),
301; Trans. ISIJ, 17 (1977), 271.

17) M. Chon and M. Tate: Tetsu-to-Hagané, 61 (1975), 948.

18) K. Polthier: Arch. Eisenhüttenw., 37 (1966), 453.

19) V. Kalinovski, M. A. Grinkov, L. Z. Khodak and V. Yu. Kaganov: Stal in Eng., (1968), 98.

20) S. Ergun: Chem. Eng. Progr., 48 (1952), 89.

21) E. Schürmann, W. Zischkale, P. Ischebeck and G. Heynert: Stal u. Eisen, 80 (1960), 854.

22) K. Polthier: Arch. Eisenhüttenw., 37 (1966), 368.

23) I. D. Semikin, G. T. Tsygankov, A. V. Borodulin, I. P. Vasko, M. S. Kruskal and V. P. Mirshabka: Izv. VUZov, Cher. Met., (1972), No. 8, 159.

24) C. A. Coberley and W. R. Marshall: Chem. Eng. Progr., 47 (1951), 141.

25) J. M. Smith: Chemical Engineering Kinetics, McGrawHill, (1956), 294. 